\title{
EPR SPECTROSCOPY STUDIES OF CHANGES IN ERYTHROCYTE MEMBRANES IN PATIENTS WITH LARYNGEAL CANCER
}

\author{
Y.B. Burlaka ${ }^{1, *}$, O.V. Sukhoveev ${ }^{2}$, N.V. Grin ${ }^{1}$, O.M. Khilchevskyi ${ }^{2}$, S.V. Verevka ${ }^{1}$ \\ ${ }^{1} S E$ "Kolomiychenko Institute of Otolaryngology of the National Academy of Medical Sciences of Ukraine”, \\ Kyiv 03057, Ukraine \\ ${ }^{2} S E$ "Institute of Bioorganic Chemistry and Petrochemistry of the National Academy of Sciences of Ukraine", \\ Kyiv 03094, Ukraine
}

\begin{abstract}
Aim: To evaluate microviscosity and sorption capacity of erythrocyte membranes (SCEM) from patients with laryngeal cancer (LC). Materials and Methods: Samples from 35 patients with LC of stages II and III and 20 healthy volunteers were investigated by electron paramagnetic resonance with Bis(1-oxyl-2,2,6,6-tetramethylpiperidinyl-4)-ester of 5,7-dimethyladamantane-1,3-dicarbonic acid (AdTEMPO) probe. SCEM was evaluated by amount of unabsorbed methylene blue. Results: Microviscosity of erythrocyte membranes was determined by the effective rotational diffusion correlation times $\left(\tau_{\mathrm{eff}}\right)$ and a decrease in radical spectrum signal intensity per hour. The most apparent decrease in mobility of the AdTEMPO in erythrocytes was observed prior to washing of erythrocytes with $0.9 \% \mathrm{NaCl}$ for 5 min after probe insertion. The deceleration after 60 min was observed only in stage II LC. $\tau_{\text {eff }}$ was at control values after washing of erythrocytes of stage II LC 5 min after probe insertion and was significantly reduced in stage III LC in comparison to control. Radical spectrum signal intensity per hour in samples of stage II and III patients prior to and after washing of erythrocytes was on average 1.5-fold higher than that of control. SCEM in samples of stage II and III LC was found in $\mathbf{4 0}$ and $33 \%$ cases, respectively and was on average significantly reduced in comparison to control. Conclusions: The initial interaction of AdTEMPO with erythrocyte membranes of stage II and III LC patients is accompanied by an increase in $\tau_{\text {eff }}$, indicating deceleration of probe rotation. $\tau_{\text {eff }}$ of the probe in membranes remains unchanged in $60 \mathrm{~min}$, indicating changes in the structural organization of lipid bilayer and its associated proteins in particular. The similarity of SCEM for both studied groups reflects the pathological changes in function of erythrocyte membranes.

Key Words: laryngeal cancer, erythrocytes, EPR, nitroxyl radicals, sorption capacity of erythrocyte membranes.
\end{abstract}

According to international epidemiological data, laryngeal cancer (LC) accounts for 30 to $40 \%$ of all malignant head and neck tumors and 1 to $2.5 \%$ of all human malignant neoplasms. In terms of histopathology, 95 to $98 \%$ of $L C$ s are of squamous cell origin. The disease is found to be more prevalent in men than women. The highest incidence of LC occurs between the fifth and seventh decade of life. Despite the constant improvements in methods for diagnosing the LC, most of diagnosed cases represent the advanced cancer (stage III-IV), which demands radical surgical approaches resulting in high rate of disability and death among the patients [1,2].

Quantitative and qualitative changes in blood content are a well-known response to exogenous and endogenous affecting factors in order to maintain homeostasis. Thus, the comprehensive study of blood cells morphology is a necessary step in the investigation of the effects that various substances exert on the organism. Erythrocytes are considered to be the universal cellular model reflecting physiological and pathological changes in the organism [3]. Considerable correlation between changes in erythrocyte membranes and plasmalemmas in organs has been established, which allows for using erythrocyte membranes as natural models for studies of permeability of all types of biomembranes [3].

Submitted: August 22, 2016.

*Correspondence: E-mail: burlakaiuliia@yahoo.com Abbreviations used: $\tau_{\text {eff }}$ - the effective rotational diffusion correlation times; AdTEMPO - Bis(1-oxyl-2,2,6,6-tetramethylpiperidinyl-4)-ester of 5,7-dimethyladamantane-1,3-dicarbonic acid; EPR - electron paramagnetic resonance; LC - laryngeal cancer; SCEM - sorption capacity of erythrocyte membranes.
Electron paramagnetic resonance (EPR) spectroscopy is a valuable technique allowing for detection of damage to cellular metabolic processes. The spin probes in particular can be used to study structural changes in cellular membranes at early stages of metabolic disruptions. A spin probe molecule is paramagnetic and can thus be investigated in the magnetic field. Nitroxyl radicals are the most widely used probes. Synthesis and study of stable nitroxyl radicals have been directed towards the creation of effective spin probes [4]. Adamantane derivatives are known to interact with natural and model membranes. They are capable of membrane integration and interaction with the lipid bilayer and integrated proteins [5]. Bis(1-oxyl-2,2,6,6-tetramethylpiperidinyl-4)-ester of 5,7-dimethyladamantane-1,3dicarbonic acid (AdTEMPO), a lipophilic nitroxyl biradical, was synthesized in Institute of Bioorganic Chemistry and Petrochemistry (Kyiv, Ukraine). It can embed in a model lipid bilayer, with nitroxyl group localizing between water and lipid phase (Fig. 1) [6]. This substance may therefore be used as a spin probe to investigate erythrocyte membrane structures. The aim of the study was to investigate microviscosity and sorption capacity of erythrocyte membranes (SCEM) from LC patients.
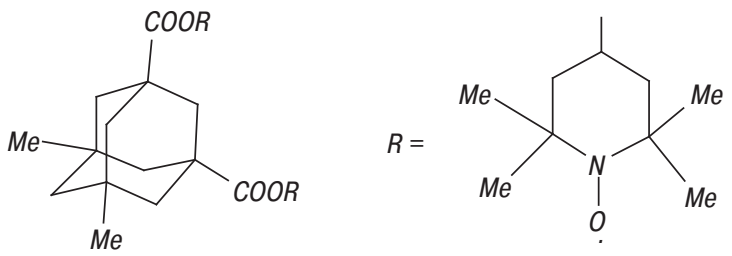

Fig. 1. Structure of the AdTEMPO/spin probe AdTEMPO 


\section{MATERIALS AND METHODS}

Patients. We investigated 35 patients of SE "Kolomiychenko Institute of Otolaryngology" (Kyiv, Ukraine). Male patients aged 45 through 65 years with initial stages of LC were selected for participation. Of them, 18 patients were diagnosed with II stage laryngeal keratotic squamous cell carcinoma (T2NOMO) and 17 with III stage (T3NOMO). The control group was composed of 20 apparently healthy volunteers. All the groups were randomized for age and sex composition. All subjects were informed about the aim of the study. Informed consent was obtained from every participant.

Biochemical measurements. Blood samples were obtained from median cubital vein puncture of fasting patients in the morning and mixed with $3.8 \%$ sodium citrate anticoagulant (9:1) in plastic test tubes. The studies were performed on erythrocytes prior to and post-triple washing with $0.9 \%$ sodium chloride solution [7]. SCEM was assayed after A.A. Togaybayev with modifications by T.V. Kopytova [8]. The amount of unabsorbed methylene blue was evaluated, and the amount of absorbed dye was calculated according to the following formula (1):

$$
A(\%)=(100-D E / D C) \cdot 100 \text {, }
$$

where $D_{E}$ is the experimental sample absorbance and $D_{c}$ is the control sample absorbance.

EPR measurements. Microviscosity of erythrocyte membranes was studied with spin probe method using adamantane-based nitroxyl radical [9]. Final probe concentration in samples was $5 \cdot 10^{-4} \mathrm{M}$. EPR spectra were registered with Varian E-3X-band spectrometer $(9 \mathrm{GHz})$. The strength of center magnetic field was $3210 \mathrm{G}$ (Gauss), a time constant $1 \mathrm{~s}$, scan time $4 \mathrm{~min}$. We used glass capillary probes of $0.1 \mathrm{ml}$ with the inner diameter of $3 \mathrm{~mm}$. The simultaneous standard signal of $\mathrm{Mn}^{2+} / \mathrm{MgO}$ ( 3 and 4 lines) with known values of $g$-factor was used to equalize experimental conditions.

We calculated the following parameters from the obtained spectra: the effective rotational diffusion correlation times $\left(\tau_{\text {eff }}\right)$, hyperfine interaction constant $(\mathrm{HI})$, and temporal change in signal intensity. Correlation time for rotational diffusion was calculated based on the following formula (2):

$$
\tau_{\text {eff }}=6,65 \cdot 10^{-10} \Delta H_{+1}\left(\sqrt{I_{+1} / I_{-1}}-1\right),
$$

where $I_{+1}, I_{-1}$ are the intensities of the low-field and high-field lines and $\Delta \mathrm{H}_{+1}$ is the peak-to-peak line width of low-field line.

Statistical processing was carried out using WinPEPI package of programs for biometrics research. Student's $t$-test and Mann - Whitney U-test were used to assess differences between the data on LC of different stages and control group. The description was made with the mean and the standard deviation for the parameters that followed a normal distribution, and the median $\left(P_{50}\right)$ and $25\left(Q_{1}\right)$ and $75\left(Q_{3}\right)$ percentiles for the variables that did not follow a normal distribution. Differences were considered significant at $p<0.05$.

\section{RESULTS AND DISCUSSION}

We measured hyperfine interaction constant from EPR spectra of the radical in erythrocyte suspension of all the groups in order to characterize spin probe environment prior to the experiments. It was measured to be $16.75 \mathrm{G}$ in all instances, which indicates that AdTEMPO embeds into the lipid bilayer of erythrocyte membrane with nitroxyl fragment situated on the lipidwater phase boundary.

The mobility of the biradical in membrane environment was expressed as effective rotational diffusion correlation time $\left(\tau_{\text {eff }}\right)$ (Table 1). Since various substances may be absorbed by cellular membranes and block their receptors, increasing their lability and disrupting permeability, all the experiments were performed on erythrocytes prior to and after washing with $0.9 \%$ sodium chloride solution. Probe diffusion was slow in samples of erythrocytes from blood of stage II and III LC patients; $T_{\text {eff }}$ at 5 min after probe insertion was $5.95 \pm 0.51 \cdot 10^{-10}$ s and $5.26 \pm 0.23 \cdot 10^{-10} \mathrm{~s}$ in comparison to $4.69 \pm 0.16 \cdot 10^{-10} \mathrm{~s}$ in the control group $(p<0.05$ and $p>0.05)$. The biradical mobility had a tendency to decrease in 60 min in stage II LC patients in comparison to control $(p>0.05)$. The difference of this parameter in stage III patients in comparison to control and the other experimental group was not significant.

Table 1. $\tau_{\text {eff }}$ in erythrocyte membranes prior to and after washing with $0.9 \%$ sodium chloride

\begin{tabular}{lcccc}
\hline \multirow{2}{*}{\multicolumn{1}{c}{ Group }} & \multicolumn{2}{c}{$\begin{array}{c}\text { Erythrocytes prior } \\
\text { to washing } \mathrm{T}_{\text {eff }} \times 10^{-10}, \mathrm{~s}\end{array}$} & \multicolumn{2}{c}{$\begin{array}{c}\text { Erythrocytes after } \\
\text { washing } \mathrm{T}_{\text {eff }} \times 10^{-10}, \mathrm{~s}\end{array}$} \\
\cline { 2 - 5 } & $5 \mathrm{~min}$ & $60 \mathrm{~min}$ & $5 \mathrm{~min}$ & $60 \mathrm{~min}$ \\
\hline Healthy volunteers & $4.69 \pm 0.16$ & $5.09 \pm 0.18$ & $4.56 \pm 0.13$ & $5.01 \pm 0.20$ \\
$(\mathrm{n}=20)$ & & & & \\
Stage II LC $(\mathrm{n}=18)$ & $5.95 \pm 0.51$ & $5.83 \pm 0.30$ & $4.18 \pm 0.69$ & $5.59 \pm 0.20$ \\
& $p<0.05$ & $p>0.05$ & $p_{1}>0.05$ & $p>0.05$ \\
Stage III LC $(\mathrm{n}=17)$ & $5.26 \pm 0.23$ & $5.62 \pm 0.22$ & $5.17 \pm 0.17$ & $5.50 \pm 0.15$ \\
& $p>0.05$ & & $p<0.02$ & \\
\hline
\end{tabular}

Note: $p$ - patient group vs healthy controls; $p_{1}$ - pre-washing $v s$ post-washing in the same group.

The value of $\tau_{\text {eff }}$ in stage II LC patients after erythrocytes washing was close to control levels 5 min after probe insertion. We observed a tendency to increased mobility of the biradical in erythrocytes after washing $\left(5.95 \pm 0.51 \cdot 10^{-10} \mathrm{~s}\right)$ in comparison to erythrocytes before washing $\left(4.18 \pm 0.69 \cdot 10^{-10} s\right)\left(p_{1}>0.05\right)$. At stage III this parameter was significantly higher than that of control $(p<0.02)$, indicating slower probe dissipation. No difference was detected between data prior to and post washing. $\tau_{\text {eff }}$ had a tendency to be higher in stage II LC patients after $60 \mathrm{~min}$ exposition $(p>0.05)$. At stage III this parameter was not significantly above that of control due to notable differences between individual readings.

Erythrocyte membrane permeability in all groups was also evaluated after the rate of radical spectrum signal drops per hour (Table 2). In stage II and III LC patients the residual signal spectrum after erythrocyte washing was on average 1.5 -fold higher than that of control ( $p<0.01$ and $p<0.001$, accordingly). After washing, this parameter in stage II and III LC patients increased statistically significantly in comparison to control in equal, although lesser measure (1.4-fold 
on average) $(p<0.02$ and $p<0.01)$. There were no significant differences between signal spectra before and after erythrocyte washing by $0.9 \%$ sodium chloride.

Table 2. EPR signal intensity of the AdTEMPO after $60 \mathrm{~min}$ in erythrocytes prior to and after triple washing with $0.9 \%$ sodium chloride

\begin{tabular}{lcc}
\hline \multicolumn{1}{c}{ Group } & $\begin{array}{c}\text { Erythrocytes prior } \\
\text { to washing, } \%\end{array}$ & $\begin{array}{c}\text { Erythrocytes after } \\
\text { washing, \% }\end{array}$ \\
\hline Healthy volunteers $(\mathrm{n}=20)$ & $47.14 \pm 2.35$ & $48.71 \pm 2.68$ \\
Stage II LC $(\mathrm{n}=17)$ & $73.00 \pm 6.11$ & $69.4 \pm 7.00$ \\
& $p<0.01$ & $p<0.02$ \\
Stage III LC $(\mathrm{n}=18)$ & $71.56 \pm 2.95$ & $66.13 \pm 4.68$ \\
& $p<0.001$ & $p<0.01$ \\
\hline
\end{tabular}

Note: $p$ - patient group $v s$ healthy controls.

EPR spectra of adamantane-based nitroxyl radical are known to be classic triplets (Fig. 2). Thus, EPR spectra of the probe after 5 min exposition do not differ from those of the control group (Fig. 2, $a, b$ ). We observed substantial difference in the control group after $60 \mathrm{~min}$. Spectrum intensity was decreased and exchange line (as result of spin - spin nitroxide interaction) appeared (Fig. 2, c). The presence of the exchange line in the control group may result from the better accessibility of membrane lipids (lipid rafts) for the lipophilic probe. Radical fragments that permeate these rafts may situate closer to each other, creating the exchange line [10]. Patient's spectra remained basically unchanged, which may signify damage to lipid bilayer structure and membrane permeability in significant fraction of the erythrocytes (Fig. 2, d).
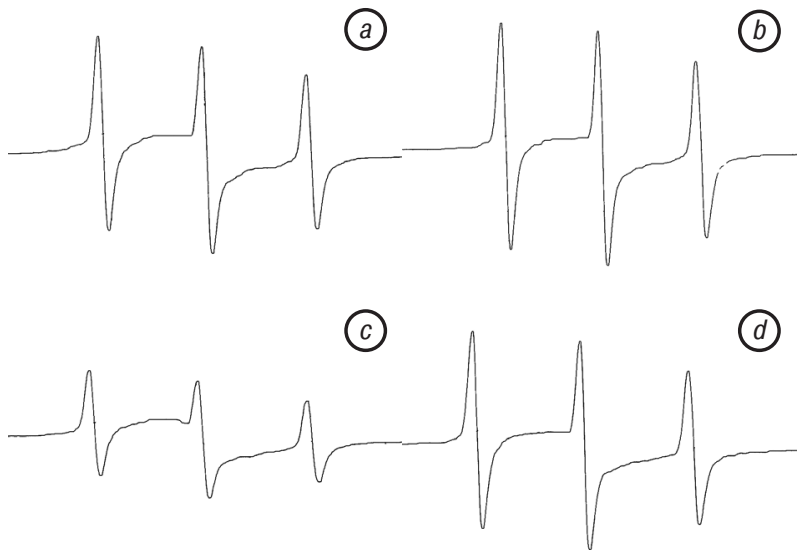

Fig. 2. The representative EPR spectra of healthy volunteers $(a, c)$ and LC patients $(b, d)$ obtained in $5(a, b)$ or $60 \min (c, d)$ after probe insertion

SCEM was detected in $40 \%$ of the LC patients with stage II and in $33 \%$ of the LC patients with stage III was on average significantly lower in comparison to the control group (Table 3). AdTEMPO, synthesized in the Institute of Bioorganic Chemistry and Petrochemistry (Kyiv, Ukraine), has been used as a paramagnetic probe to investigate interactions with membranes of plastids and subplastid systems as well as mixed phospholipid liposomes from sunflower, soybean and egg yolk $[6,9,11]$. We were the first group to use it to assess microviscosity of erythrocyte membranes in LC patients. The probe diffusion may be interpreted as happening in two stages: membrane surface sorption and permeation into lipid bilayer. We can thus observe using EPR spectra various $\tau_{\text {eff }}$ for binding of AdTEMPO to membrane surface ( $5 \mathrm{~min}$ ) and during the probe permeation of erythrocyte membranes (60 min). Primary sorption of AdTEMPO on erythrocyte membranes is associated with increased $\tau_{\text {eff }}$, which indicates inhibited torsion of the probe. The effective rotational diffusion correlation time further increased in $60 \mathrm{~min}$, yet these changes are not substantial, which may result from disruptions in lipid bilayer structure and integrated proteins [12]. This assumption was confirmed by data of residual signal intensity. Cellular membrane permeability decreases significantly in LC patients, and this impedes intracellular antioxidants from interacting with nitroxyl radical that is on the outer surface of the plasmalemma. The cellular membrane of the healthy volunteers is intact and functions normally. The presence of the exchange line in the control group may be associated with better availability of lipid rafts to the lipophilic probe. Radical fragments in them are situated closer to each other [10].

Table 3. SCEM in LC patients and healthy controls

\begin{tabular}{lc}
\hline \multicolumn{1}{c}{ Group } & Mean; $\mathrm{P}_{50} ; \mathrm{Q} 1-\mathrm{Q} 3$ \\
\hline Healthy volunteers $(\mathrm{n}=20)$ & $45.89 ; 50.21(43.85 ; 53.68)$ \\
Stage II LC $(\mathrm{n}=10)$ & $2.35 ; 0.92(0.4 ; 4.3)^{\star}$ \\
Stage III LC $(\mathrm{n}=11)$ & $2.03 ; 1.63(0.23 ; 4.22)^{\star}$ \\
\hline
\end{tabular}

Note: *difference between patient group and healthy controls is significant, $p<0.05$.

Evaluation of sorption capacity is widely applied to assess a state of erythrocyte membranes. SCEM analysis provides information on reduction capacity of erythrocytes, which changes with fluctuation in plasmallemma's barrier functions. Diminished sorption capacity is interpreted by some authors as an indicator of energy deficiency in the erythrocyte. Thus, degradation of erythrocyte membranes and transmembrane transport leads to disruptions in energy metabolism within the erythrocytes accompanied by decreased ATP content and pyruvate kinase activity [13]. The increased SCEM is perceived as an indicator of their damage and cellular degradation [8] .

Erythrocyte membrane permeability has been shown to increase under recurrent infections of upper respiratory tract, chronic obstructive pulmonary disease, dermatoses, acute pancreatitis, chronic nephropathies, etc., depending on the gravity and stage of the pathological conditions [8, 14-16]. Decreased SCEM was observed only in patients with the metabolic syndrome [17]. To the best of our knowledge, there are no studies on SCEM in cancer.

Hence, we established in the present study the decrease in SCEM from blood of LC patients in comparison to healthy volunteers, irrespectively of the stage of the disease. Notably, there is no significant difference between patients of the both experimental groups. We consider the decrease of SCEM in blood of the LC patients as an indicator of pathological changes in surface functional activity of erythrocyte membranes, in particular of their inability to efficiently transport metabolites via bloodstream $[13,18]$. 


\section{REFERENCES}

1. Siegel RL, Miller KD, Jemal A. Cancer statistics, 2016. CA Cancer J Clin 2016; 66: 7-30.

2. Cancer of the Head and Neck. Shah JP, Patel SG, eds. Hamilton, ON: BC Decker Inc, 2001. 487 p.

3. Novitskii VV, Ryazantseva NV, Stepanova EA. Physiology and Pathophysiology of the Erythrocyte. Tomsk: TGU, 2004. 202 p. (in Russian).

4. Lamoureux G, Artavia G. Use of the adamantane structure in medicinal chemistry. Curr Med Chem 2010; 17: 2967-78.

5. Morse PD, Lusczakoski-Nesbitt DM, Clarkson RB. Adamantyl nitroxide: A spin label for probing membrane surfaces. Chem Phys Lipids 1982; 31: 257-73.

6. Melnyk AK, Samus N, Hilchevsky OM, et al. Nitroxyl biradicals on adamantane platform. Rep Nat Acad Sci Ukraine 2012; (4): 138-44 (in Ukrainian).

7. Laboratory Methods of Research in the Clinic: Directory. Menshikov VV, ed. Moscow: Medicine, 1987. 368 p. (in Russian).

8. Kopytova TV. Study of the sorption capacity of red blood cell membranes for the assessment of the pattern of endogenous intoxication in dermatoses. Klin Lab Diagn 2006; (1); 18-9 (in Russian).

9. Melnyk AK, Sukhoveev OV, Kononets LA, et al. An EPR spin probe study of liposomes from sunflower and soybean phospholipids. J Liposome Res 2016; 26: 80-6.

10. Grammenos A, Mouithys-Mickalad PH, Guelluy $\mathrm{M}$, et al. ESR technique for noninvasive way to quantify cyclodextrins effect on cell membranes. Biochem Biophys Res Commun 2010; 398: 350-4.
11. Kochubey SM, Vovk AI, Bondarenko OYu, et al. Heterogeneity of thylakoid membranes studied by EPR spin probe. Biochemistry (Mosc) 2007; 72: 558-64.

12. Kartel MT, Ivanov LV, Lyapunov OM, et al. Estimation of the effect of carbon nanotubes on the microviscosity of erythrocyte membranes by the spin probe method. Rep Nat Acad Sci Ukraine 2015; (3): 114-21 (in Russian).

13. Novitskii VV, Stepovaya EA, Batukhtin AV, et al. Erythrocyte membranes in patients with malignant tumors as shown by fluorescent probing. Bull Exp Biol Med 1999; 128: $857-9$.

14. Muravlyova LE, Molotov-Luchanskiy VB, Kluyev DA, et al. Metabolic status of erythrocytes at patients with chronic obstructive pulmonary disease. Archiv Euromedica 2013; 3:44-6.

15. May JM, Qu ZC, Cobb CE. Reduction and uptake of methylene blue by human erythrocytes. Am J Physiol Cell Physiol 2004; 286: C1390-8.

16. Gavrilyuk VP, Nazarenko PM, Konoplya AI. Structurally functional disturbances of erythrocytes and their correction in patients with the mild and severe acute pancreatitis. Kursk Sci Pract Bull «Man and his Health» 2007; (3): 29-36 (in Russian).

17. Atyasova ES. The study of endogenous intoxication in the blood of patients with metabolic syndrome. Med Almanac 2009; (1) [cited 2016 Aug 19]. Available from: http://www.medalmanac.ru/ ru/old/archive/year2009/ session/partticipients/1668 (in Russian).

18. Mikhailovich VA, Marusanov VE, Bichun AB, Domanskaia IA. Erythrocyte membrane permeability and the sorption capacity of erythrocytes - optimal criteria of the severity of endogenous intoxication. Anesteziol Reanimatol 1993; (5): 66-9 (in Russian). 